\title{
Discovery of small molecule inhibitors of huntingtin exon 1 aggregation by FRET- based high-throughput screening in living cells
}

Chih Hung Lo ${ }^{1}$, Nitin K. Pandey ${ }^{2}$, Colin Kin-Wye Lim ${ }^{1}$, Zhipeng Ding ${ }^{1}$, Meixin Tao ${ }^{2}$, David D. Thomas $^{3-4}$, Ralf Langen ${ }^{2}$ and Jonathan N. Sachs ${ }^{1 *}$

${ }^{1}$ Department of Biomedical Engineering, University of Minnesota, Minneapolis, MN 55455

${ }^{2}$ Department of Physiology and Neuroscience, Zilkha Neurogenetic Institute, Keck School of Medicine, University of Southern California, Los Angeles, CA 90033

${ }^{3}$ Department of Biochemistry, Molecular Biology and Biophysics, University of Minnesota, Minneapolis, MN 55455

${ }^{4}$ Photonic Pharma LLC, Minneapolis, MN 55410

*To whom correspondence should be addressed: jnsachs@umn.edu 


\section{Supporting information}
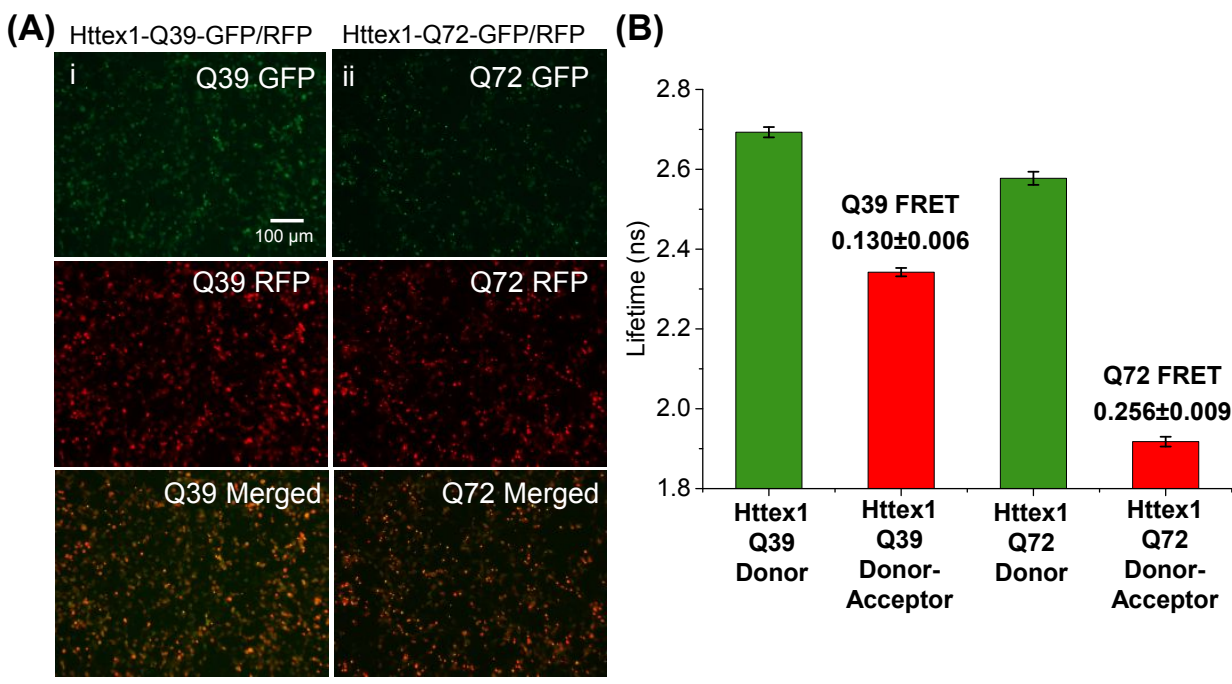

Supplementary Fig. 1: Characterization of Httex1 intermolecular FRET biosensors. (A) Fluorescent microscopy images of GFP and RFP-tagged Httex1 of two different Q-lengths, (i) Q39 and (ii) Q72, expressed in HEK293 cells. Puncta are observed in Httex1-Q39 FRET biosensor and more distinctively and greater in number in Httex1-Q72 FRET biosensor. (B) Fluorescence lifetime measurements of the Httex1Q39 and Httex1-Q72 FRET biosensors. Data are means \pm SD of three independent experiments. 


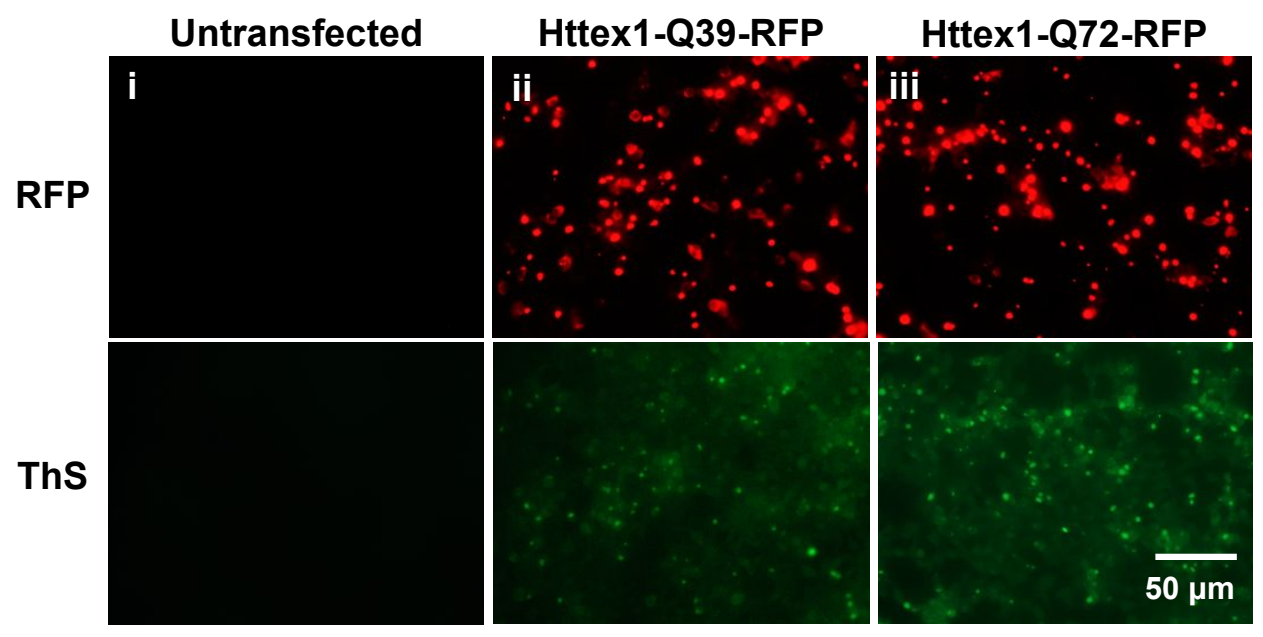

Supplementary Fig. 2: Thioflavin-S (ThS) staining of HEK293 cells expressing Httex1-Q39-RFP or Httex1-Q72-RFP. Fluorescence images of ThS staining of HEK293 cells being (i) untransfected or transfected with (ii) Httex1-Q39-RFP or (iii) Httex1-Q72-RFP. The amount of Httex1-RFP plasmids transfected for ThS assay is equal to the total amount of Httex1-GFP and Httex1-RFP plasmids used in the FRET biosensors. Httex1-GFP is not used because it will interfere with the ThS signal which is green in fluorescence. Images are representative of three independent experiments. 

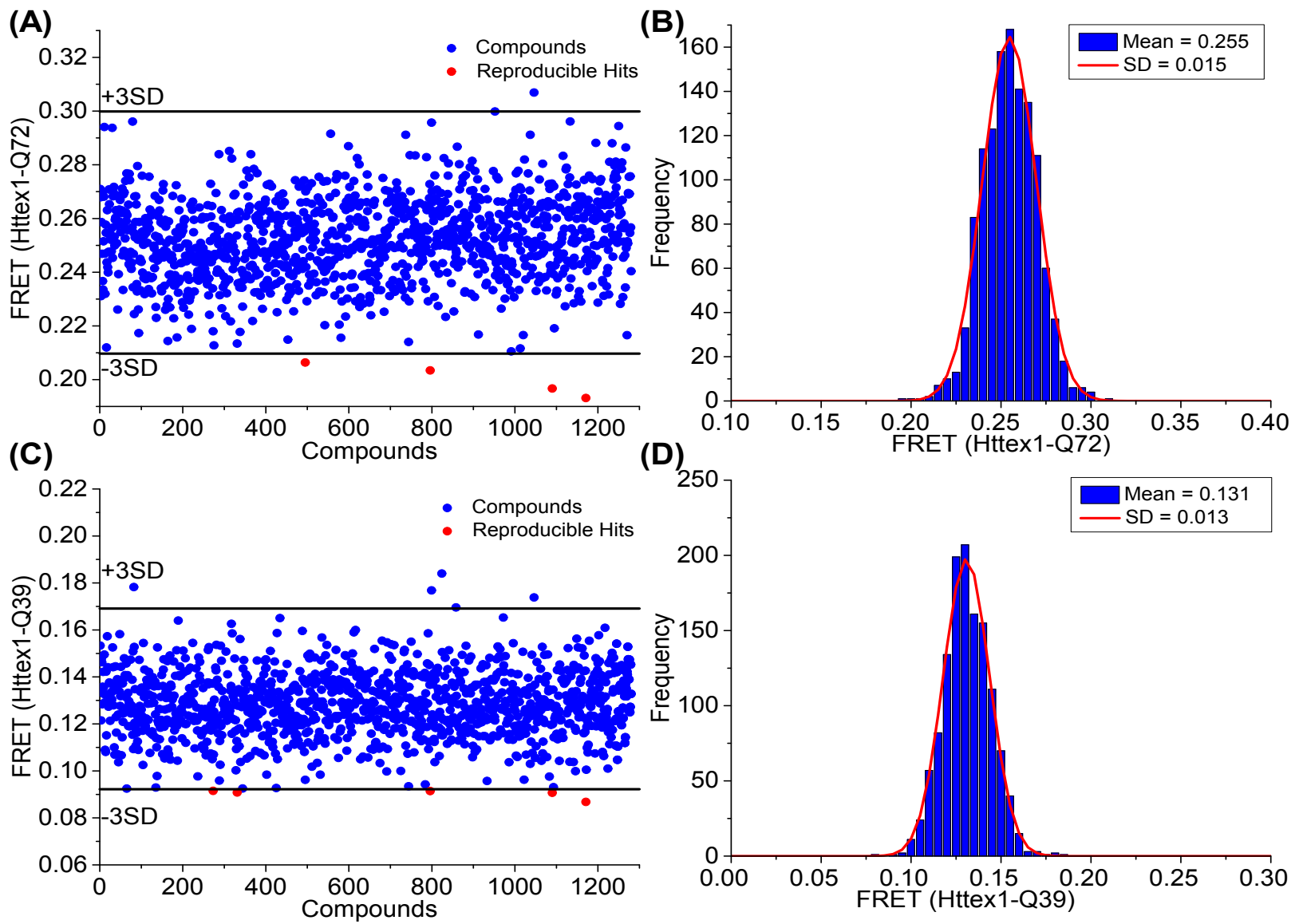

(E)

\begin{tabular}{|c|c|c|}
\hline \multicolumn{3}{|c|}{ Hits with dose-dependent FRET reduction } \\
\hline Q72-Hits & Q39-Hits & Compound name \\
\hline 1171 & 1171 & Niclosamide \\
\hline- & 331 & $10058-F 4$ \\
\hline 796 & 796 & AMG 9810 \\
\hline 495 & - & TNP \\
\hline 1090 & 1090 & TBB \\
\hline- & 273 & DMS \\
\hline
\end{tabular}

Supplementary Fig. 3: High-throughput screening (HTS) of LOPAC library using Httex1-Q72 and Httex1-Q39 FRET biosensors and identification of hit compounds. Representative pilot screening plots and Gaussian fits of the LOPAC library containing 1280 compounds screened using (A-B) Httex1-Q72 and (C-D) Httex1-Q39 FRET biosensors. A FRET efficiency cutoff threshold was applied at a change in FRET efficiency of 3SD (black lines). Reproducible hits that decreased FRET by more than 3SD below the mean of all cells were highlighted in red. Gaussian fits were performed by plotting histograms of all compounds from the LOPAC screens with the Httex1 FRET biosensors after removal of fluorescent compounds to obtain the average FRET efficiency and the standard deviation (SD) of the screens. (E) Hits from both screens are summarized. Screening plots are representative of three independent experiments. 
(A)

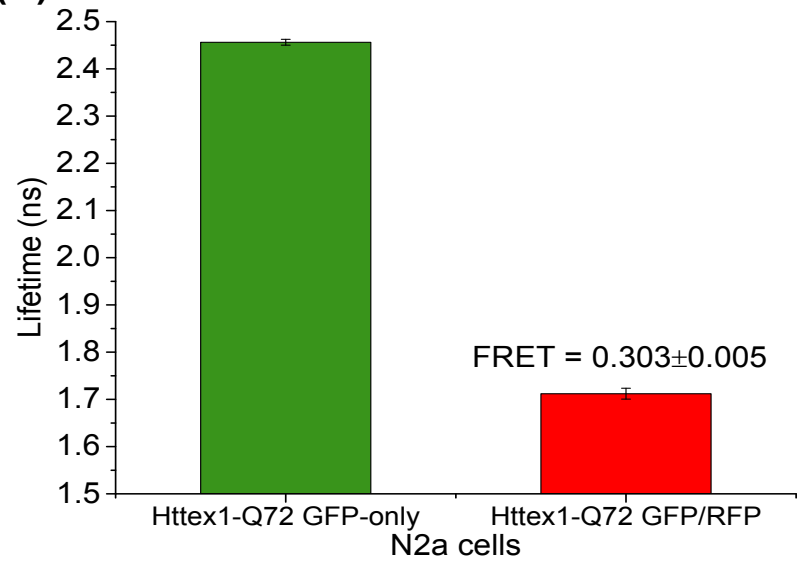

(B)

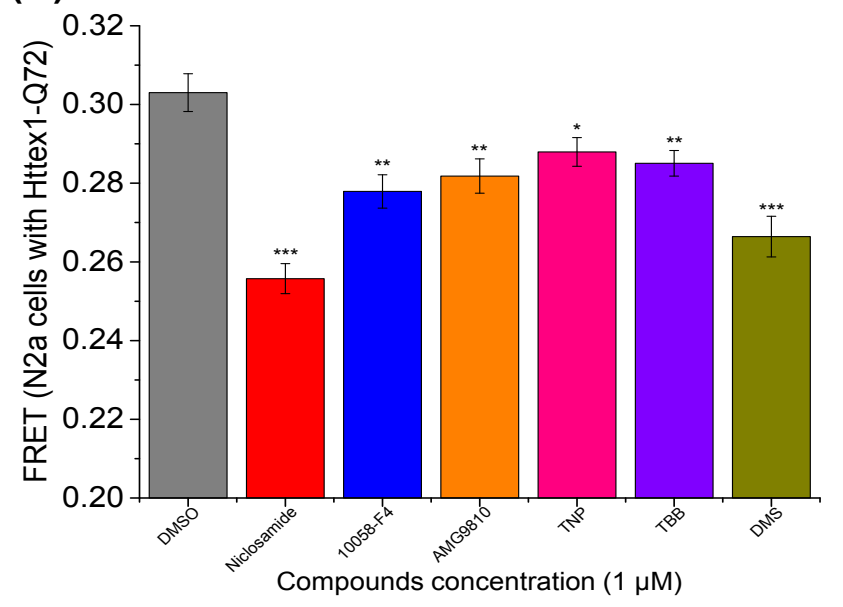

Supplementary Fig. 4: Hit compounds reduced FRET in the Httex1-Q72 biosensor expressed in N2a cells. (A) Fluorescence lifetime measurements of the Httex1-Q72 FRET biosensor in N2a cells. (B) Hit compounds $(1 \mu \mathrm{M})$ reduced FRET in the Httex1-Q72 biosensor expressed in N2a cells. Data are means \pm SD of three independent experiments. ${ }^{*} P<0.05, * * P<0.01$, and $* * * P<0.001$ by two-tailed unpaired $t$ test. 

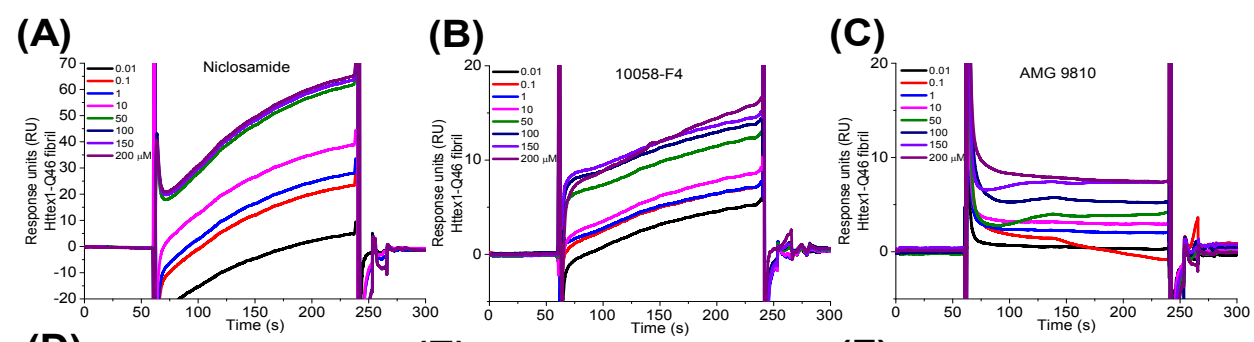

(D)

(E)
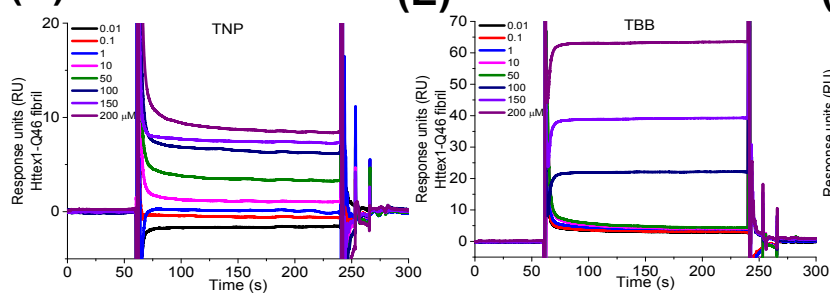

$(\mathbf{F})$

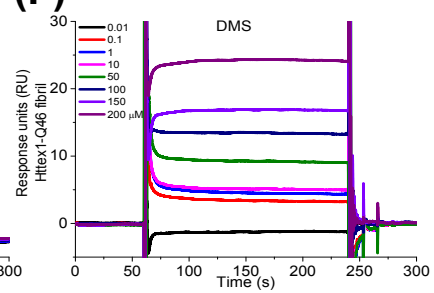

Supplementary Fig. 5: Binding of the hit compounds to Httex1-Q46 fibrils as characterized by SPR measurements. SPR raw binding curves for (A) niclosamide, (B) 10058-F4, (C) AMG9810, (D) TNP, (E) $\mathrm{TBB}$, and (F) DMS. Bind curves are representative of three independent experiments. 
(A)

35R1-Control

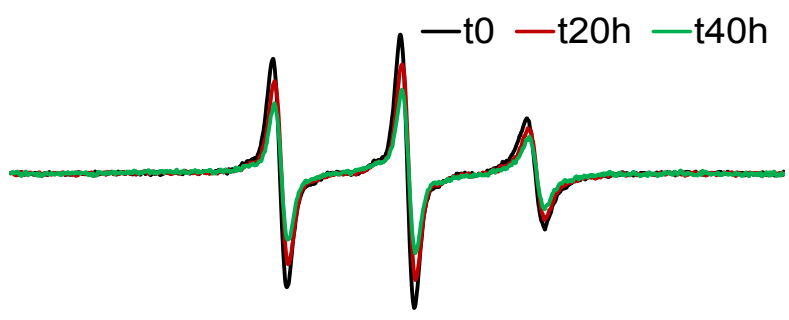

(C)

$35 \mathrm{R} 1+2 \%$ Seed

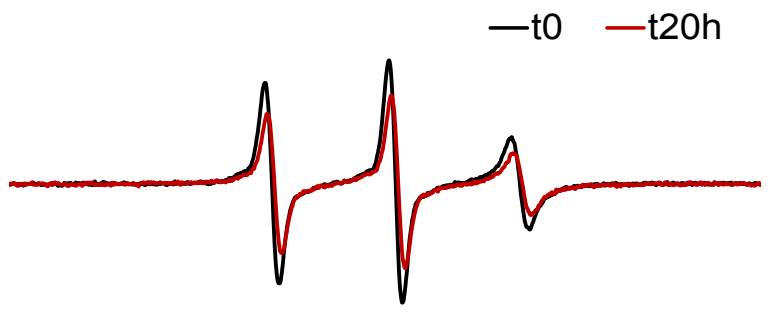

(B)

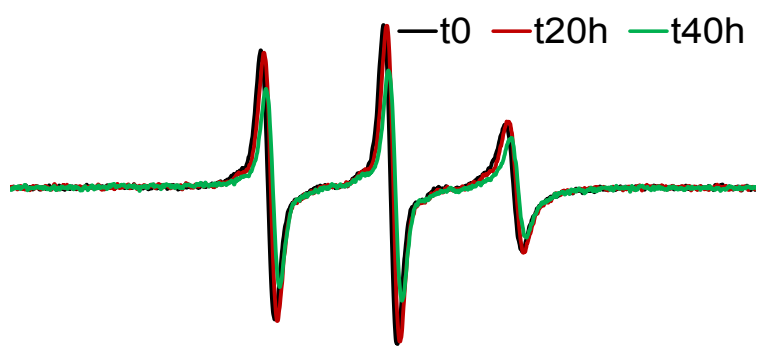

(D)

$35 \mathrm{R} 1+2 \%$ Seed + Niclosamide

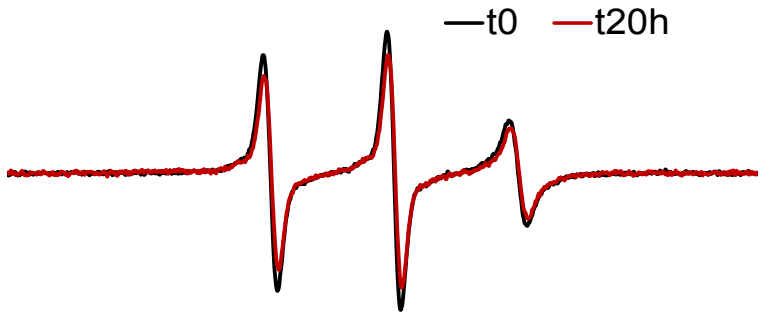

Supplementary Fig. 6: Inhibition of Httex1-Q46 aggregation by hit compounds as characterized by continuous wave (CW)-electron paramagnetic resonance (EPR) measurements. (A-B) EPR characterization of the inhibition of Httex1-Q46 aggregation in the absence of seed (unseeded) and treated with (A) DMSO only as control or (B) hit compounds (niclosamide is shown as a representative compound at $20 \mu \mathrm{M}$ ). (C-D) Inhibition of Httex1-Q46 aggregation with $2 \%$ seed added to the proteins and treated with (C) DMSO only as control or (D) hit compounds (niclosamide is shown as a representative compound at 20 $\mu \mathrm{M})$. Httex1-Q46 was spin labeled at position 35 and the hit compounds were treated for 20 or 40 hours. Aggregation or misfolding causes line broadening and a concomitant reduction in amplitude and inhibition by hit compounds revert these observations. EPR raw curves are representative of three independent experiments. 